\section{Bem-estar psicológico de jovens de 18 a 24 anos: fatores associados}

\author{
Psychological well-being of young people 18 to \\ 24 years of age and associated factors
}

\section{Abstract}

This study aimed to identify factors associated with psychological well-being among young people 18 to 24 years of age in Pelotas, Rio Grande do Sul State, Brazil. In a population-based crosssectional study, 1,621 subjects answered a structured questionnaire on socio-demographic data, religion, employment, and substance use, as well as the Mini International Neuropsychiatric Interview (MINI) to assess psychiatric disorders. The Faces Scale (Andrews) was used to evaluate psychological well-being. Poisson regression was used for multivariate analysis. Of the total sample, $85.3 \%$ displayed psychological well-being, which was positively associated with non-use of illicit drugs, current employment, religion, socioeconomic status (classes A and B), higher educational levels, and absence of psychiatric disorders. Programs to reduce poverty and encourage education and identification and prevention of drug use among youths are of paramount importance to improve their health and psychological well-being.

Personal Satisfaction; Mental Disorders; Street Drugs; Young Adult
Luciano Dias de Mattos Souza ${ }^{1}$ Tauana da Costa Maragalhoni 1 Maiara Tavares Quincoses ${ }^{1}$ Karen Jansen 1 Ana Luara Sica Cruzeiro 1 Liliane Ores 1 Ricardo Azevedo da Silva 1 Ricardo Tavares Pinheiro 1

\section{Introdução}

O bem-estar constitui um campo de estudos que procura compreender as avaliações que as pessoas fazem de suas vidas 1 . Essa área de investigações teve um crescimento acelerado na última década, revelando como seus principais tópicos de pesquisa a satisfação e a felicidade. $\mathrm{O}$ constructo bem-estar se desdobra em um componente cognitivo chamado de satisfação com a vida, e um componente afetivo chamado felicidade. O componente afetivo se subdivide, por sua vez, em uma tendência da pessoa a experienciar sensações positivas (afeto positivo) e uma tendência a perceber sensações negativas (afeto negativo). Essas duas disposições são independentes. Afetividades negativas e positivas não se referem apenas à frequência de tais experiências, mas também a sua intensidade 2 .

Os estudos existentes em nosso país sobre a saúde de jovens dificilmente estendem um olhar sob a perspectiva do bem-estar. Portanto, julgase importante conhecer melhor as percepções dos jovens com relação a ele. Ainda há a necessidade de aprofundar estudos e buscar mais dados a respeito dessa percepção que possam contribuir para intervenções e formulação de políticas públicas para essa faixa etária. No fim da adolescência e início da vida adulta, o jovem se defronta com uma série de tarefas psicológicas, entre as quais se destacam a separação psicológica em 
relação aos progenitores, a construção da autonomia e a consolidação da autoestima ${ }^{3}$, o que pode gerar algum tipo de sofrimento psíquico associado ao baixo nível de bem-estar.

Entre os eventos estressantes capazes de influenciar a sensação de bem-estar psicológico estão a morte de uma pessoa importante para o indivíduo, desemprego, separação conjugal, bem como roubo e doenças na família 4 . A religiosidade está associada aos níveis mais elevados de bem-estar. Pessoas que praticam semanalmente apresentam maiores níveis de bem-estar psicológico do que as que praticam esporadicamente ou nunca 5. Os menores níveis de bem-estar psicológico estão associados a duas variáveis: o baixo nível socioeconômico e a baixa escolaridade dos pais do jovem. Em relação aos comportamentos de saúde, como: sedentarismo, tabagismo e a ingestão de bebidas alcoólicas estão associados a baixo nível de bem-estar. Já o uso de drogas ilícitas não apresenta associação com bem-estar psicológico, provavelmente por um problema de aferição ${ }^{5}$. Dessa forma, a presente investigação tem como objetivo identificar os fatores associados ao bem-estar psicológico, dentro de uma amostra de jovens de 18 a 24 anos da cidade de Pelotas, Rio Grande do Sul, Brasil.

\section{Método}

Delineamento

Trata-se de um estudo quantitativo seccional de base populacional.

\section{Amostra}

O universo em estudo corresponde à população de 39.667 jovens (Instituto Brasileiro de Geografia e Estatística.Cidades@.http://www.ibge.gov.br/ cidadesat/topwindow.htm?1, acessado em 31/ Mai/2008) na faixa etária compreendida entre os 18 e 24 anos de idade, residentes nos 448 setores censitários da zona urbana da cidade de Pelotas.

Para tal universo, foi calculada a amostra para verificação da prevalência de bem-estar psicológico, estimando-se a prevalência de $72 \% 5 \mathrm{com}$ erro de $2.5 \%$ e intervalo de $95 \%$ de confiança. Tal cálculo foi realizado com uso do programa Epi Info 6.04d (Centers for Disease Control and Prevention, Atlanta, Estados Unidos). Assim, seria necessária a participação de 1.202 indivíduos. A esse número foram acrescidos $30 \%$ para controle de fatores de confusão, perdas e recusas. Consequentemente, a amostra necessária para aferição da prevalência de bem-estar psicológico no referido universo de jovens entre 18 e 24 anos da cidade de Pelotas foi de 1.562 indivíduos. Tendo em vista que o presente projeto se insere em uma investigação de maior porte, o número amostral da presente pesquisa foi superior em virtude de outras variáveis de interesse em estudo.

O desenho amostral adotado foi de amostragem por conglomerados. Posteriormente, foram sorteados 89 setores para a identificação dos jovens que participarão da investigação. A seleção dos domicílios nos setores sorteados foi realizada segundo uma amostragem sistemática, sendo o primeiro domicílio a residência da esquina pré-estabelecida pelo Instituto Brasileiro de Geografia e Estatística (IBGE) como início do setor, o intervalo de seleção foi determinado por um pulo de dois domicílios entre os sorteados. Os aplicadores foram orientados a seguir o percurso da contagem dos domicílios aleatoriamente em qualquer uma das ruas pertencentes ao setor censitário em questão, respeitando-se o intervalo de seleção previamente estabelecido (duas casas). Portanto, pretendeu-se assegurar a representatividade amostral da presente investigação. Quando encontrado mais de um jovem que preenchia os critérios para participação no estudo na mesma residência, eles foram convidados para realização da investigação.

Foram excluídos da amostra os jovens, identificados pela equipe de pesquisa, que residiam na zona não urbana de Pelotas ou que apresentam dificuldade em compreender e/ou responder ao questionário.

\section{Instrumentos}

Para a coleta das informações utilizadas na presente pesquisa, foi feita uma entrevista estruturada que contém questões referentes à saúde e ao comportamento dos jovens. O aplicador seguiu um questionário que aborda itens como: sexo, idade, estado civil, cor, escolaridade, moradia, atividade laboral, religiosidade, história familiar, comportamento no trânsito, comportamentos violentos, condições de saúde, sexualidade e outros comportamentos de risco à saúde.

Nível socioeconômico foi investigado em uma pergunta, com base na Escala de Indicadores de Classe Social da Associação Brasileira das Empresas de Pesquisa (Critério de classificação econômica Brasil. http://www.abep.org/codigosguias/ CCEB2008Base2006e2007.pdf.2006, acessado em 20/Jun/2009), que indica quais e quantos eletrodomésticos a pessoa possui em sua residência e a escolaridade do chefe da família.

O trabalho foi aferido por meio de uma pergunta com objetivo de identificar se o entrevistado havia sido remunerado em algum emprego nos últimos 12 meses (sim/não). 
Foi questionada a escolaridade do entrevistado, sendo divididas as respostas em 4 categorias: de 0 a 4 anos de estudo, de 5 a 8 anos, de 9 a 11 anos e 12 ou mais anos de estudo.

Religiosidade foi investigada com perguntas sobre a crença atual, frequência de prática em sua religião e se pertence a algum grupo religioso.

Quanto a exercício físico, indagou-se se o jovem realizou algum tipo de atividade física ou se é sedentário.

O item alcoolismo, tabagismo e uso de drogas ilícitas foi investigado por questões baseadas na Alcohol, Smoking and Substance Involvement Screening Test (ASSIST) 6, que procura saber se a pessoa já usou alguma substância, a frequência do uso, se sente desejo, se tem amigos ou familiares que utilizam algum tipo de substância.

Transtornos psiquiátricos foram avaliados por entrevista diagnóstica padronizada breve Mini Internacional Neuropsychiatric Interview 5.0 (MINI) 7. Essa entrevista é de curta duração - 15 a 30 minutos -, destinada à utilização na prática clínica e de pesquisa, que visa à classificação diagnóstica de forma compatível com os critérios do Manual Diagnóstico e Estatístico de Transtornos Mentais - 4a edição (DSM-IV), compreendida de 19 módulos que avaliam 17 transtornos do eixo I do DSM-IV, risco de suicídio e transtorno de personalidade antissocial. Se o jovem apresentar qualquer um dos transtornos aferidos, será considerado presença de transtorno psiquiátrico.

O bem-estar psicológico foi avaliado mediante a Escala de Faces de Andrews ${ }^{8}$, em que cada figura da escala consiste em um círculo, com olhos que não mudam, e uma boca que varia de um meio círculo voltado para cima até outro meio círculo semelhante voltado para baixo. O jovem assinalou aquela figura que mais se assemelhava à maneira como ele se sentia a respeito da sua vida no último mês. $\mathrm{O}$ bem-estar psicológico foi considerado quando o jovem marcou uma das três faces com semicírculo voltado para cima; as demais respostas foram avaliadas como ausência de bem-estar. Embora não exista estudo de validação para a população brasileira, a escala tem sido utilizada de forma ampla 4,5,9. Por se tratar de um instrumento visual analógico, ele é facilmente compreendido universalmente, sendo utilizado também em populações infantis 10,11.

\section{Equipe de pesquisa, seleção e treinamento de entrevistadores}

Foram selecionados acadêmicos do Centro de Ciências da Vida e da Saúde da Universidade Católica de Pelotas (UCPel) mediante a avaliação de currículo acadêmico e experiência prévia de participação em investigações científicas. A equipe recebeu treinamento para homogeneizar os procedimentos de abordagem das residências, assinatura do Termo de Consentimento após esclarecimentos sobre o estudo para os jovens identificados e aplicação dos instrumentos e entrevista.

\section{Processamento e análise de dados}

O programa Epi Info 6.04d foi utilizado para dupla digitação dos dados e checagem automática da amplitude e consistência. As análises estatísticas foram realizadas por meio dos programas Stata 9.0 (Stata Corp., College Station, Estados Unidos) e SPSS 13.0 (SPSS Inc., Chicago, Estados Unidos). Para análise dos dados, inicialmente foi realizada a descrição da amostra por intermédio das frequências simples e percentuais de cada variável em estudo. A análise bivariada ocorreu com os testes qui-quadrado e t de Student com os quais foram verificadas as associações do bemestar psicológico e de todas as variáveis independentes em estudo. Na análise multivariada, foi utilizada a regressão de Poisson para avaliar a razão de prevalência das variáveis independentes em relação ao desfecho. A referida análise foi controlada para efeito de conglomerado (setor censitário do participante). O nível de significância pré-estabelecido foi de $\mathrm{p}<0,050$.

\section{Aspectos éticos}

Neste protocolo de pesquisa, foram respeitados todos os princípios éticos estabelecidos pelo Conselho Nacional de Saúde (Resolução $n^{o}$. 196/1996). Os jovens receberam informações sobre os objetivos da pesquisa e assinaram o Termo de Consentimento Livre e Esclarecido. O projeto foi aprovado pelo Comitê de Ética em Pesquisa da UCPel.

\section{Resultados}

A Tabela 1 mostra as características da amostra estudada. Dos 1.621 entrevistados, mais da metade são do sexo feminino, grande parcela da amostra possui classificação socioeconômica C e a proporção de pessoas que têm de 9 a 11 anos de estudo foi de $41 \%$. Com relação ao uso de substâncias, $73,2 \%$ bebeu alguma bebida alcoólica nos últimos três meses, enquanto 31,7\% e $12,8 \%$ fizeram, respectivamente, uso de tabaco e drogas ilícitas durnate o mesmo período. $\mathrm{Na}$ amostra de jovens, ainda pode ser observado que $32,3 \%$ frequentam missa/culto/sessão religiosa pelo menos uma vez por mês, a maioria dos jovens trabalhou nos últimos 12 meses 
Caracterização dos jovens de 18 a 24 anos. Pelotas, Rio Grande do Sul, Brasil.

\begin{tabular}{|c|c|c|}
\hline Variáveis & n & $\%$ \\
\hline \multicolumn{3}{|l|}{ Sexo } \\
\hline Feminino & 904 & 55,8 \\
\hline Masculino & 717 & 44,2 \\
\hline \multicolumn{3}{|l|}{ Escolaridade (anos) } \\
\hline $0-4$ & 118 & 7,3 \\
\hline $5-8$ & 356 & 22,0 \\
\hline $9-11$ & 662 & 41,0 \\
\hline 12 ou mais & 479 & 29,7 \\
\hline \multicolumn{3}{|l|}{ Drogas } \\
\hline Não & 1.377 & 87,2 \\
\hline Sim & 203 & 12,8 \\
\hline \multicolumn{3}{|c|}{ Bebeu álcool nos últimos três meses } \\
\hline Não & 430 & 26,8 \\
\hline Sim & 1.173 & 73,2 \\
\hline \multicolumn{3}{|l|}{ Fumou nos últimos três meses } \\
\hline Não & 1.095 & 68,3 \\
\hline Sim & 508 & 31,7 \\
\hline \multicolumn{3}{|l|}{ Pratica religião } \\
\hline Não & 1.092 & 67,7 \\
\hline Sim & 522 & 32,3 \\
\hline \multicolumn{3}{|l|}{ Classificação socioeconômica } \\
\hline A ou $B$ & 608 & 37,5 \\
\hline C & 780 & 48,1 \\
\hline D ou $E$ & 232 & 14,3 \\
\hline \multicolumn{3}{|l|}{ Trabalhou nos últimos 12 meses } \\
\hline Não & 530 & 33,1 \\
\hline Sim & 1.070 & 66,9 \\
\hline \multicolumn{3}{|l|}{ Bem-estar psicológico } \\
\hline Não indicativo de bem-estar & 238 & 14,7 \\
\hline Indicativo de bem-estar & 1.382 & 85,3 \\
\hline
\end{tabular}

e $85,3 \%$ dos jovens pontuou um indicativo de bem-estar psicológico.

$\mathrm{Na}$ análise bivariada (Tabela 2), as variáveis que estiveram associadas ao bem-estar psicológico foram o sexo feminino, classificação socioeconômica A ou B, elevada escolaridade, o trabalho no último ano, o uso de drogas e de tabaco nos últimos três meses, bem como a presença de transtorno psiquiátrico.

$\mathrm{Na}$ análise multivariada (Tabela 2), o bemestar psicológico teve associação significativa nas seguintes variáveis: a classificação socioeconômica A ou B, elevada escolaridade, trabalho remunerado no último ano, prática de religião, uso de drogas nos últimos três meses e presença de transtorno psiquiátrico. Verificou-se na variável baixo nível socioeconômico que aqueles adolescentes cuja família pertencia ao nível socioeconômico D ou E apresentaram $11 \%$ menos chances de ter relatado bem-estar psicológico em relação aos jovens de famílias de nível socioeconômico A ou B. Da mesma forma, a elevada escolaridade esteve diretamente associada à proporção de bem-estar psicológicoo. Quanto maior a escolaridade, maior a proporção de bem-estar no grupo de jovens pesquisados ( $\mathrm{p}<0,050$ ). $\mathrm{O}$ uso de drogas nos últimos três meses também esteve associado com uma menor proporção de bem-estar psicológico ( $\mathrm{RP}=0,92$; IC95\%: 0,85$0,99)$. Os que relataram algum tipo de prática religiosa tiveram probabilidade de relatar o bemestar psicológico 5\% maior. A mesma magnitude pode ser encontrada na comparação entre jovens que trabalharam de forma remunerada no 
Modelo hierárquico final para bem-estar psicológico por regressão de Poisson. Pelotas, Rio Grande do Sul, Brasil, 2007-2009.

\begin{tabular}{|c|c|c|c|c|}
\hline Variável & RP bruta (IC95\%) & Valor de $p$ & RP ajustada (IC95\%) & Valor de $\mathrm{p}$ \\
\hline Sexo * & & 0,030 & & 0,538 \\
\hline Masculino & $1,05(1,00-1,09)$ & & $1,01(0,97-1,05)$ & \\
\hline Feminino & Referência & & Referência & \\
\hline Nível socioeconômico ** & & $<0,001$ & & $<0,001$ \\
\hline$A$ ou $B$ & Referência & & Referência & \\
\hline C & $0,93(0,90-0,97)$ & & $0,96(0,92-1,00)$ & \\
\hline D ou $E$ & $0,83(0,77-0,90)$ & & $0,89(0,83-0,97)$ & \\
\hline Escolaridade do jovem (anos) & & $<0,001$ & & $<0,001$ \\
\hline Até 4 & Referência & & Referência & \\
\hline $5-8$ & $1,08(0,96-1,22)$ & & $1,08(0,96-1,20)$ & \\
\hline $9-11$ & $1,17(1,04-1,30)$ & & $1,12(1,00-1,24)$ & \\
\hline 12 ou mais & $1,24(1,11-1,39)$ & & $1,13(1,02-1,26)$ & \\
\hline Trabalho remunerado no último ano & & 0,010 & & 0,030 \\
\hline Sim & $1,06(1,01-1,11)$ & & $1,05(1,01-1,10)$ & \\
\hline Não & Referência & & Referência & \\
\hline Pratica religião & & 0,107 & & 0,009 \\
\hline Sim & $1,03(0,99-1,08)$ & & $1,05(1,01-1,09)$ & \\
\hline Não & Referência & & Referência & \\
\hline Uso de drogas nos últimos três meses & & $<0,001$ & & 0,041 \\
\hline Sim & $0,87(0,80-0,94)$ & & $0,92(0,85-0,99)$ & \\
\hline Não & Referência & & Referência & \\
\hline Uso de bebida alcoólica nos últimos três meses * & & 0,136 & & 0,675 \\
\hline Sim & $1,04(0,99-1,09)$ & & $1,01(0,97-1,06)$ & \\
\hline Não & Referência & & Referência & \\
\hline Fumou nos últimos três meses * & & 0,008 & & 0,608 \\
\hline Sim & $0,94(0,90-0,98)$ & & $1,01(0,97-1,06)$ & \\
\hline Não & Referência & & Referência & \\
\hline Presença de transtorno psiquiátrico & & $<0,001$ & & $<0,001$ \\
\hline $\operatorname{Sim}$ & $0,72(0,68-0,77)$ & & $0,75(0,70-0,80)$ & \\
\hline Não & Referência & & Referência & \\
\hline
\end{tabular}

IC95\%: intervalo de 95\% de confiança; RP: razão de prevalência.

* Variável retirada do modelo hierárquico na análise ajustada;

** Variável não controlada na análise ajustada.

último ano e aqueles que não o fizeram. Os portadores de transtorno mental têm menor chance de indicar bem-estar psicológico (RP: 0,75; IC95\%: 0,70-0,80). Portanto, a associação do uso de tabaco com o bem-estar psicológico não foi mais estatisticamente significativa.

\section{Discussão}

No presente estudo a grande maioria dos jovens entrevistados assinalou as expressões correspondentes ao bem-estar, indicando uma avaliação positiva de sua vida no último mês. Em uma pes- quisa de base populacional com pessoas maiores de vintes anos, utilizando o mesmo instrumento 4 encontrou uma prevalência de mal-estar psicológico de $14 \%$. Da mesma forma, Silva et al. 5 apontaram a ocorrência de bem-estar em 89,6\% dos adolescentes.

Entre os jovens investigados neste estudo, o sentimento de bem-estar psicológico é influenciado pelo nível socioeconômico e a escolaridade, o que constatou maior prevalência em jovens que se encontram em um nível socioeconômico mais alto e aqueles que têm maior escolaridade. Tal resultado é semelhante ao de um trabalho sobre bem-estar psicológico e adolescência 5 . 
O nível socioeconômico está relacionado a um melhor status funcional de saúde ${ }^{12}$. Esse aspecto se vincula a uma melhor percepção de bem-estar e maior capacidade funcional, o que pode ter refletido no desempenho acadêmico dos jovens de Pelotas.

Em relação à religiosidade, permaneceram as associações entre maior bem-estar psicológico e frequente prática religiosa. Aqueles que relataram praticar sua religiosidade mostraram maior chance de satisfação com a vida quando comparados com os que declararam pouca frequência ou nenhuma religiosidade. Sabe-se que indivíduos com indicativo de transtorno psiquiátrico têm menores escores de bem-estar psicológico 13 e, em estudantes, cada vez mais existem evidências que associam percepção subjetiva de bemestar do sujeito em relação à sua crença religiosa com suas condições de saúde mental ${ }^{14}$. Logo, a presença de transtornos psiquiátricos, a prática religiosa e o bem-estar psicológico parecem ser conceitos de mútua interação.

Quanto ao trabalho, jovens que relataram ter recebido algum tipo de remuneração no último ano, apresentam maior nível de bem-estar psicológico do que os que não receberam nenhum tipo de remuneração. Segundo Siqueira \& Padovam 1, a satisfação no trabalho tem sido apontada como um vínculo afetivo positivo e tem sido definida como aspecto específico desse vínculo às satisfações advindas do salário pago. Locke (1976, apud Siqueira \& Padovam ${ }^{1}$ ) diz que um trabalho ou experiência de trabalho pode resultar em um estado emocional positivo ou de prazer.

No que tange ao uso de substâncias, os resultados mostram que o uso de drogas ilícitas se relaciona de maneira inversa com o bem-estar psicológico. O abuso de álcool diminuiu significativamente a proporção de percepção de bemestar psicológico 5 . Tal discrepância pode ser explicada pela ampla sensibilidade utilizada na presente investigação que aferiu uso de álcool nos últimos três meses. Outra diferença de resul- tados pode ser observada entre esses estudos. Na presente pesquisa, o não uso de drogas ilícitas esteve significativamente associado ao bem-estar psicológico, relação não observada em Silva et al. ${ }^{5}$. Essa diferença pode ser explicada pela faixa etária dos dois estudos e pela maior prevalência de uso de substâncias ilícitas no grupo de jovens de 18 a 24 anos.

A utilização de uma escala visual para avaliação do bem-estar psicológico registra sentimentos inspirados pela experiência diária e garante que todos os jovens sejam estimulados da mesma forma, o que torna essa categoria de escala um método válido e confiável na aferição de bem-estar psicológico entre jovens. Apesar disso, foram encontrados poucos estudos na literatura científica que empregam tal instrumento, ou mesmo que investigam esse tema. A impossibilidade de afirmar qualquer tipo de relação causal entre o bem-estar psicológico e as variáveis independentes em estudo também limitam a interpretação de seus achados. Contudo, a presente pesquisa trata de uma investigação com amostra representativa de jovens que busca novas informações a respeito de um tema negligenciado.

Assim, constatou-se a associação entre bemestar psicológico com baixo nível socioeconômico e baixa escolaridade, indicando que os jovens de classes sociais menos favorecidas apresentam menores índices de bem-estar psicológico. Dessa forma, programas que visem à redução da pobreza, incentivo à educação, identificação e prevenção de uso de drogas em jovens são de extrema importância para a melhoria do bem-estar psicológico e prevenção da saúde. Estudos mostram que programas que visam à melhora do bemestar subjetivo, implicam diretamente sobre os conceitos que as pessoas têm de sua qualidade de vida e sobre sua saúde. Entre esses programas, pode-se destacar a Psicologia Positivista que se preocupa com a promoção da saúde e aspectos saudáveis e adaptativos do desenvolvimento 15. 


\section{Resumo}

Esta investigação visou identificar os fatores associados ao bem-estar psicológico em jovens de 18 a 24 anos de Pelotas, Rio Grande do Sul, Brasil. Em estudo transversal de base populacional, 1.621 jovens responderam a um questionário estruturado com questões referentes a dados sociodemográficos, prática de religião, trabalho remunerado, uso de substâncias e o Mini International Neuropsychiatric Interview (MINI) para avaliação dos transtornos psiquiátricos. A Escala de Faces de Andrews avaliou o bem-estar psicológico. A regressão de Poisson foi utilizada para análise multivariada. Dos entrevistados, 85,3\% apresentaram bem-estar psicológico, que esteve associado ao não uso de drogas ilícitas, ao trabalho remunerado, à prática de religião, às classes socioeconômicas A e B, à alta escolaridade e a não presença de transtornos psiquiátricos. Programas que visem à redução da pobreza, incentivo à educação, identificação e prevenção de uso de drogas em jovens são de extrema importância para a melhoria do bemestar psicológico e prevenção da saúde.

Satisfação Pessoal; Transtornos Mentais; Drogas Ilícitas; Adulto Jovem

\section{Colaboradores}

L. D. M. Souza participou da concepção do projeto, redação do artigo, revisão crítica da literatura relevante do conteúdo intelectual, análise e interpretação dos dados, assim como da aprovação final da versão a ser publicada. T. C. Maragalhoni e M. T. Quincoses participaram da redação do artigo, revisão crítica da literatura relevante do conteúdo intelectual, análise e interpretação dos dados, e aprovação final da versão a ser publicada. K. Jansen participou da concepção do projeto, redação do artigo e da aprovação final da versão a ser publicada. A. L. S. Cruzeiro e L. Ores participaram da concepção do projeto e da aprovação final da versão a ser publicada. R. A. Silva participou da concepção do projeto, redação do artigo, revisão crítica da literatura relevante do conteúdo intelectual e da aprovação final da versão a ser publicada. R. T. Pinheiro participou da concepção do projeto, redação do artigo, análise e interpretação dos dados e da aprovação final da versão a ser publicada.

\section{Agradecimentos}

Ao Conselho Nacional de Desenvolvimento Científico e Tecnológico.

\section{Referências}

1. Siqueira MMM, Padovam VAR. Bases teóricas de bem-estar subjetivo, bem-estar psicológico e bemestar no trabalho. Psicol Teor Pesq 2008; 24:201-9.

2. Gouveia VV, Fonsêca PN, Lins SLB, Lima AV, Gouveia RSV. Escala de bem-estar afetivo no trabalho (Jaws): evidências de validade fatorial e consistência interna. Psicol Reflex Crít 2008; 21:464-73.
3. Dias GF, Fontaine AM. Tarefas desenvolvimentais e bem-estar dos jovens: algumas implicações para o aconselhamento psicológico. Cadernos de Consulta Psicológica 1996; (12):103-14.

4. Sparrenberger F, Santos I, Lima RC. Associação de eventos de vida produtores de estresse e mal-estar psicológico: um estudo de base populacional. Cad Saúde Pública 2004; 20:249-58. 
5. Silva RA, Horta BL, Pontes LM, Faria AD, Souza LDM, Cruzeiro ALS, et al. Bem-estar psicológico e adolescência: fatores associados. Cad Saúde Pública $2007 ; 23: 1113-8$.

6. Henrique IFS, De Micheli D, Lacerda RB, Lacerda LA, Formigoni MLOS. Validação da versão brasileira do teste de triagem do envolvimento com álcool, cigarro e outras substâncias (ASSIST). Rev Assoc Med Bras 2004; 50:199-206.

7. Amorim P. Mini Internacional Neuropsychiatric Interview (MINI): validação de entrevista breve para diagnóstico de transtornos mentais. Rev Bras Psiquiatr 2000; 22:106-15.

8. McDowell I, Newell C. Measuring health: a guide to rating scales and questionnaires. New York: Oxford University Press; 1996.

9. Hallal PC, Dumith SC, Bertoldi AD, Scalco DL, Menezes AMB, Araújo CL. Well-being in adolescents: the 11-year follow-up of the 1993 Pelotas (Brazil) birth cohort study. Cad Saúde Pública 2010; 26:1887-94.

10. Veronese G, Castiglioni M, Tombolani M, Said M. 'My happiness is the refugee camp, my future Palestine': optimism, life satisfaction and perceived happiness in a group of Palestinian children. Scand J Caring Sci 2011; http://onlinelibrary.wiley.com/ doi/10.1111/j.1471-6712.2011.00951.x/abstract.
11. Cardoso CMP. Estratégias de coping, bem-estar e adaptação nas crianças e adolescentes com cancro: estudo exploratório [Dissertação de Mestrado]. Lisboa: Universidade de Lisboa; 2010.

12. Sun S, Chen J, Johannesson M, Kind P, Xu L, Zhang Y, et al. Population health status in China: EQ-5D results, by age, sex and socio-economic status, from the National Health Services Survey 2008. Qual Life Res 2011; 20:309-20.

13. Marques LF. A saúde e o bem-estar espiritual em adultos porto-alegrenses. Psicol Ciênc Prof 2003; 23:56-65.

14. Gastaud MB, Souza LDM, Braga L, Horta CL, Oliveira FVM, Sousa PLR, et al. Bem-estar espiritual e transtornos psiquiátricos menores em estudantes de psicologia: estudo transversal. Rev Psiquiatr Rio Gd Sul 2006; 28:12-8.

15. Scorsolini-Comin F, Santos MA. The scientific study of happiness and health promotion: an integrative literature review. Rev Latinoam Enferm $2010 ; 18: 472-9$.

Recebido em 16/Dez/2010

Versão final reapresentada em 14/Fev/2012 Aprovado em 28/Fev/2012 